\title{
Colorectal cancer is a leading cause of cancer incidence and mortality among adults younger than 50 years in the USA: a SEER-based analysis with comparison to other young-onset cancers
}

\author{
Abhishek Bhandari, ${ }^{1}$ Melissa Woodhouse, ${ }^{2}$ Samir Gupta ${ }^{3}$
}

- Additional material is published online only. To view please visit the journal online (http://dx.doi.org/10. 1136/jim-2016-000229).

${ }^{1}$ Department of Hospital Medicine, Northwestern University, Northwestern Memorial Hospital, Chicago, Illinois, USA

${ }^{2}$ Beckman Research Institute of the City of Hope, Duarte, California, USA

${ }^{3}$ Department of Medicine, Division of Gastroenterology, Veterans Affairs San Diego Health Care System, and the Moores Cancer Center University of California, San Diego, California, USA

\section{Correspondence to} Dr Samir Gupta, San Diego Veterans Affairs Healthcare System, 3350 La Jolla Village Drive MC 111D, San Diego, CA 92161, USA; s1gupta@ucsd.edu

Accepted 24 October 2016 Published Online First 18 November 2016

Copyright (C) 2016 American Federation for Medical Research

\begin{abstract}
Colorectal cancer (CRC) incidence and mortality are rising among young adults. Our aim was to contrast the relative incidence and mortality of CRC to other common cancers among young adults in the USA. We used Surveillance, Epidemiology, and End Results registry data to compare cancer site-specific and age-specific mortality and incident rates for adults younger than age 50 . We summarized extracted data, both overall, and stratified by sex. We found CRC was the third leading cause of cancer death among adults younger than age 50, after breast and lung cancer ( 1.67 cases per $100,000)$. Among young women, CRC was the fourth leading cause of cancer death (1.51 per $100,000)$. Among young men, CRC was the second leading cause of cancer death (1.82 cases per $100,000)$. CRC was the second most incident cancer among young adults for men and women combined. Among men, CRC was the second most incident cancer after age 30 , with $4.9,9.0,16.4$, and 30.8 cases per 100,000 for ages 30-34, 35-39, 40-44, and 45-49 years, respectively. Among women, CRC incidence was similar with 4.2, 7.6, 15.3, and 25.9 cases per 100,000 for ages 30-34, 35-39, 40-44, and $45-49$ years, respectively. These results show that $C R C$ is a leading cause of cancer incidence and mortality among young adults in the USA, relative to other cancers. Given trends toward increasing rates of CRC among young adults, strategies for identifying individuals at risk for young-onset CRC who might benefit from early age of screening initiation merit investigation.
\end{abstract}

\section{INTRODUCTION}

Colorectal cancer (CRC) is a leading cause of cancer death worldwide, accounting for 693,900 deaths in 2012. ${ }^{1}$ Largely as a result of screening, overall CRC incidence and mortality in adults over the age of 50 have been declining over the past 30 years. $^{2}$ However, CRC incidence and mortality are rising among adults younger than 50 in the USA. ${ }^{2-4}$ For example, data from the US National Cancer Database 1998-2007 suggest an overall annual percentage increase per year of $2.7 \%$ for colon, and $3.9 \%$ for rectal cancer among adults younger than $50 .^{4}$ The median age for young-onset

\section{Significance of this study}

What is already known about this subject?

- Colorectal cancer (CRC) is a leading cause of cancer death worldwide.

- Incidence and mortality of CRC among young adults has been increasing.

- CRC cases in young adults are more likely to undergo delayed diagnoses and have advanced stages.

What are the new findings?

- Colorectal cancer (CRC) was the third leading cause of cancer death among adults younger than age 50 in the USA.

- CRC was the second leading cause of cancer death among men younger than age 50 and the fourth leading cause of cancer death among women younger than age 50.

- CRC was the second most incident cancer death among adults younger than age 50 in the USA.

- The incidence of CRC especially increased in young adults over the age of 30 .

\section{How might these results change the focus of research or clinical practice? \\ - Given the burden of colorectal cancer (CRC) on young adults, strategies for identifying individuals at risk for young-onset CRC who might benefit from early age of screening initiation merit investigation.}

CRC reported was 44 years, with $75 \%$ of cases occurring between age 40 and 49 . Young-onset cases are more likely to be associated with delayed diagnosis, as well as advanced stage disease. These observations have led to a call for heightened awareness of early signs and symptoms of CRC that should promote diagnostic work up among young adults, identification of risk factors associated with young-onset CRC, and even consideration of earlier age of screening initiation. ${ }^{4}$ 
However, assessment of public health priorities for screening research and interventions requires understanding absolute rates of CRC incidence and mortality, along with an assessment of CRC incidence and mortality relative to other cancer types. The aim of this study was to use population-based cancer registry data to contrast CRC incidence and mortality relative to other common cancers, overall, and by sex.

\section{METHODS}

We used the US Surveillance, Epidemiology, and End Results (SEER) registry to obtain CRC incidence and mortality data for the most common cancers among adults younger than 50 years, 2001-2010, using the SEER Cancer Query system. The US National Cancer Institute (NCI) oversees the SEER program, which collects and publishes cancer incidence and survival data from population-based cancer registries covering $\sim 28 \%$ of the US population. ${ }^{6}$ The SEER registry comprises data from SEER 18 database, which includes the following states and metropolitan areas: Atlanta, greater California, Connecticut, Detroit, greater Georgia, rural Georgia, Hawaii, Iowa, Kentucky, Los Angeles, Louisiana, New Jersey, New Mexico, San Francisco-Oakland, San Jose-Monterey, Seattle-Puget Sound, Utah, and the Alaska Native Tumor Registry. ${ }^{6}$

There were two primary objectives of the study: first, we compared cancer site-specific mortality rates across cancer types. In order to achieve this, we obtained age-adjusted cancer mortality rates per 100,000 for common cancers affecting individuals aged 20-44 years (mortality data through age 49 were not readily available for analyses). Second, we compared cancer site-specific incident rates. To accomplish this, we extracted age-specific incidence per 100,000 for common cancers for individuals aged 2049 years. We used descriptive statistics to summarize data, overall, and stratified by sex and cancer site (colon vs rectum); all analyses were performed using Microsoft Excel.

\section{RESULTS}

CRC mortality, relative to other cancer types among young adults

We found that CRC was the third leading cause of cancer death among young adults age 20-45 years, after breast and lung cancer, at 1.67 cases per 100,000 (figure 1). Among young women, CRC was the fourth leading cause of cancer death, at 1.51 per 100,000 , behind breast, lung, and cervical cancer, respectively (figure 2). For young men, CRC was the second leading cause of cancer death, at 1.82 cases per 100,000, second only to lung cancer (figure 3). When stratified by location (colon and rectum) as well as gender, rectal and in particular colon cancer remained common young-onset cancers (see online supplementary appendix tables $1-3$ ).

\section{CRC incidence, relative to other cancer types among young adults}

We found that CRC was the second most incident cancer among young adults for men and women combined (figure 4). Among young men, CRC became the second most incident cancer after age 30, with 4.9, 9.0, 16.4, and 30.8 cases per 100,000 for ages $30-34,35-39,40-44$, and 45-49 years, respectively (figure 5). Among young women, CRC incidence patterns were similar to young men after age 30 , with $4.2,7.6,15.3$, and 25.9 cases per 100,000 for ages 30-34, 35-39, 40-44, and 45-49 years, respectively (figure 6). Notably, among young women, CRC incidence was dwarfed by breast cancer incidence overall, and less distinct from other cancers when compared with that seen in young men. When stratified by location (colon and

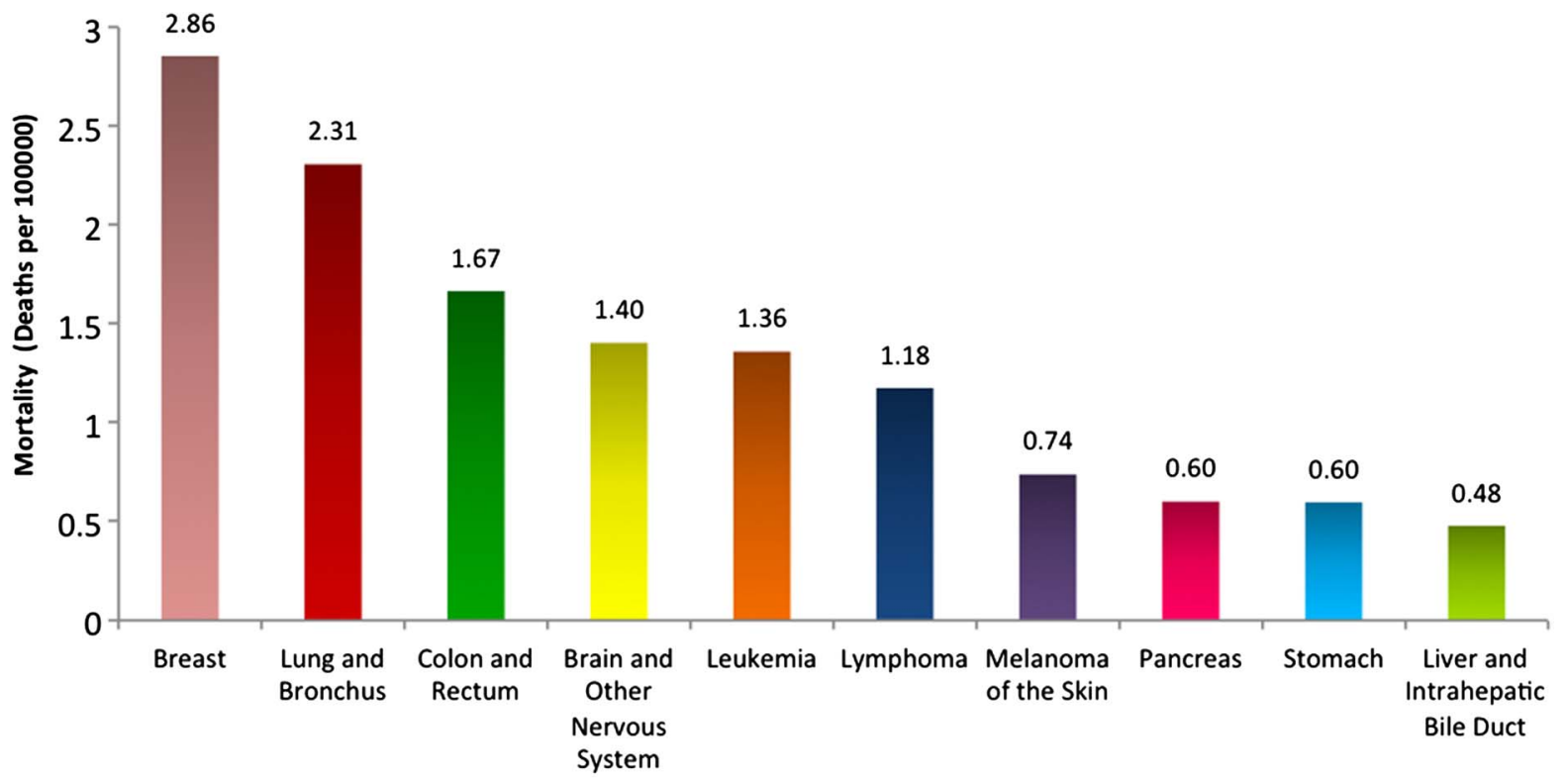

\section{Cancer Type}

Figure 1 Age-adjusted mortality for the 10 most common cancers among young men and women combined, age 20-44 years, SEER 2001-2010. 


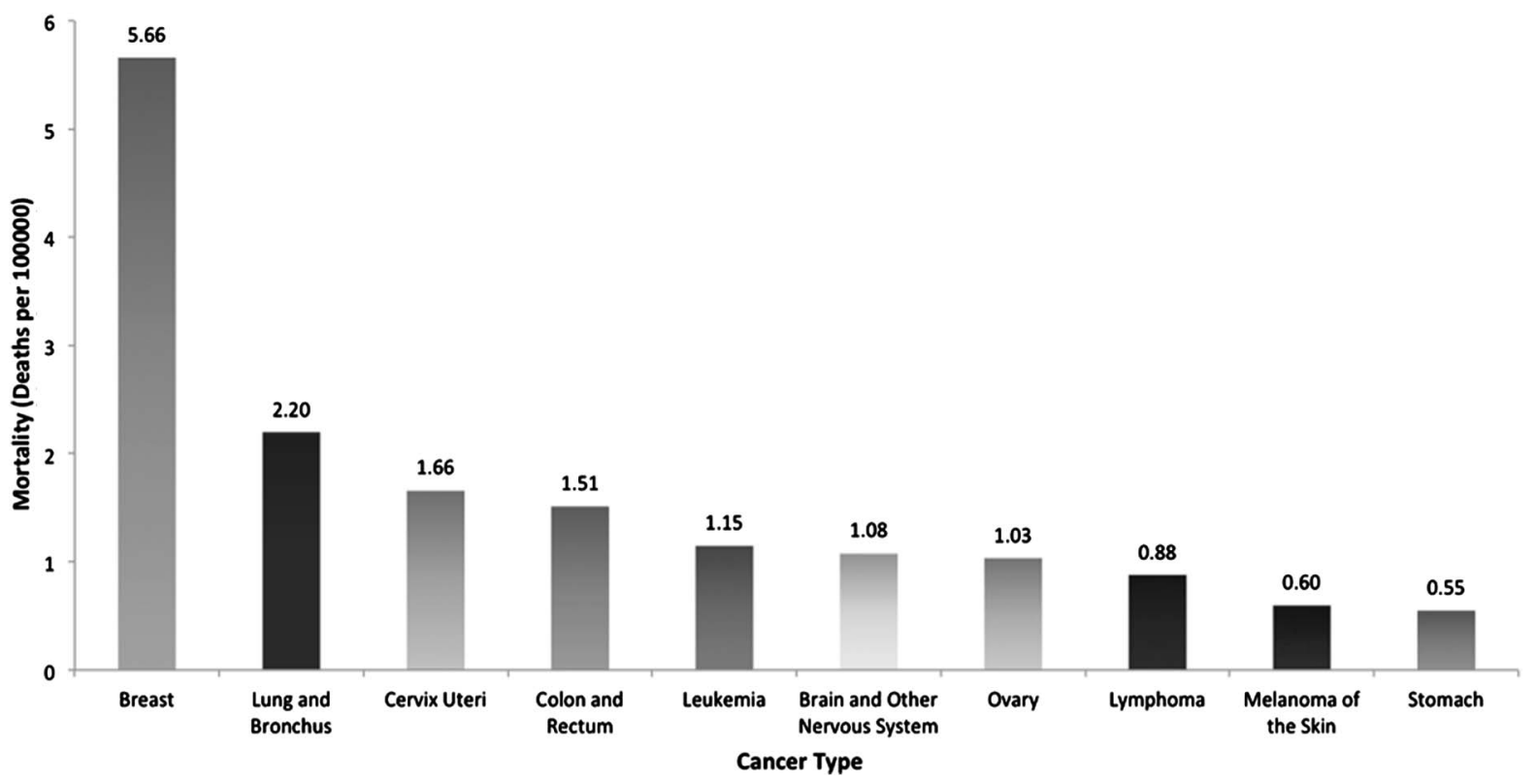

Figure 2 Age-adjusted mortality of the 10 most common cancers among young women, age 20-44 years, SEER 2001-2010.

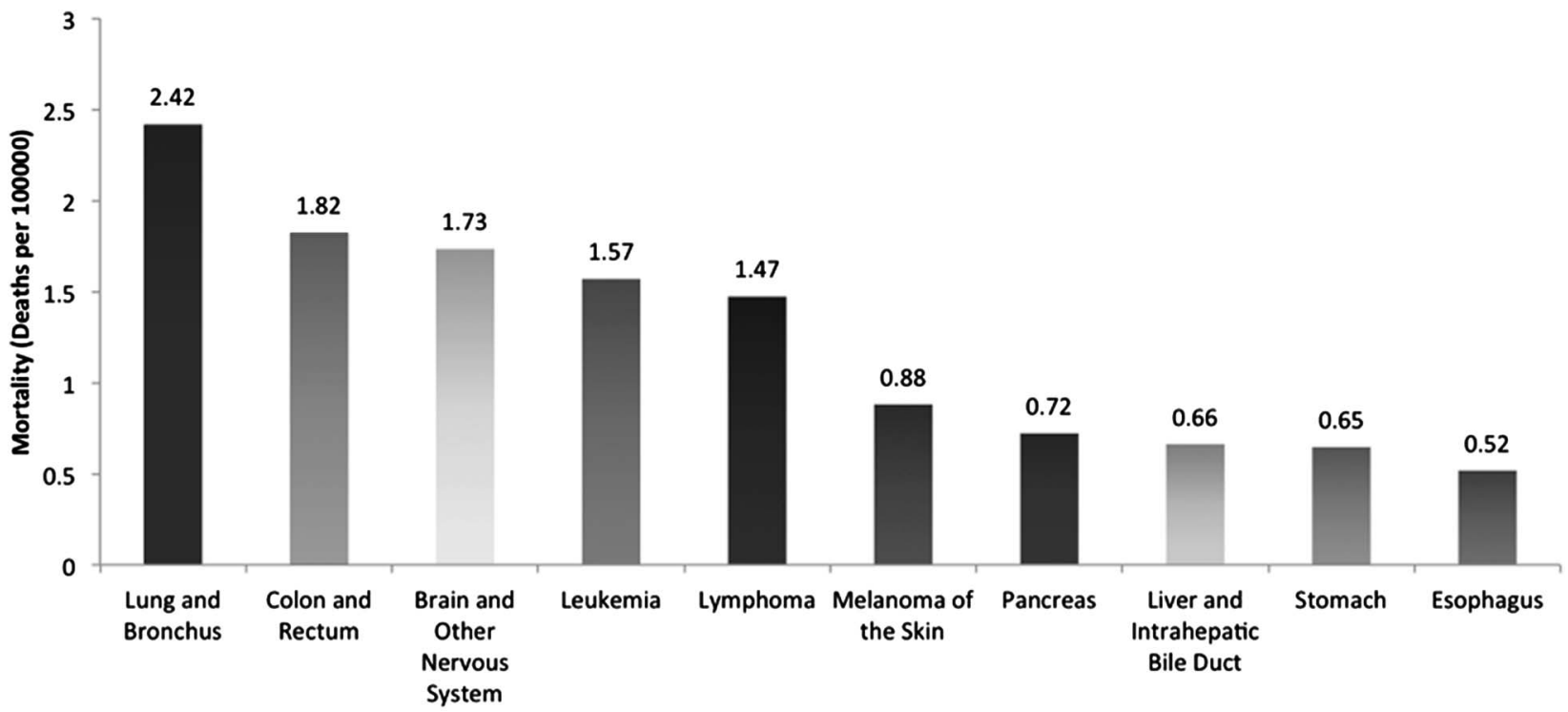

Cancer Type

Figure 3 Age-adjusted mortality of the 10 most common cancers among young men, age 20-44 years, SEER 2001-2010.

rectum) as well as gender, rectal as well as colon cancer remained common contributors to young-onset cancer incidence (see online supplementary appendix tables 4-6).

\section{DISCUSSION}

As is true among individuals age 50 and older, we found CRC was a leading cause of cancer incidence and mortality, relative to other cancers in the USA. CRC is unique among common cancers affecting young adults, in that there exist a number of effective (fecal immunochemical testing, colonoscopy, sigmoidoscopy) and emerging (CT colonography, multitarget stool DNA testing) screening tests available that could be considered for implementation. Taken together with knowledge of the rising rate of CRC among young adults, observed higher stage of presentation associated with young-onset disease, and availability of proven and emerging screening tests for CRC, we believe these observations support a call to action for policymakers, public health officials, clinicians, and researchers to develop and support initiatives to identify individuals younger than age 50 for CRC screening. While we agree that heightened attention to potential signs and symptoms of CRC (such as rectal bleeding or iron deficiency anemia), and implementation of family-history-based guidelines for screening is 


\section{Original research}

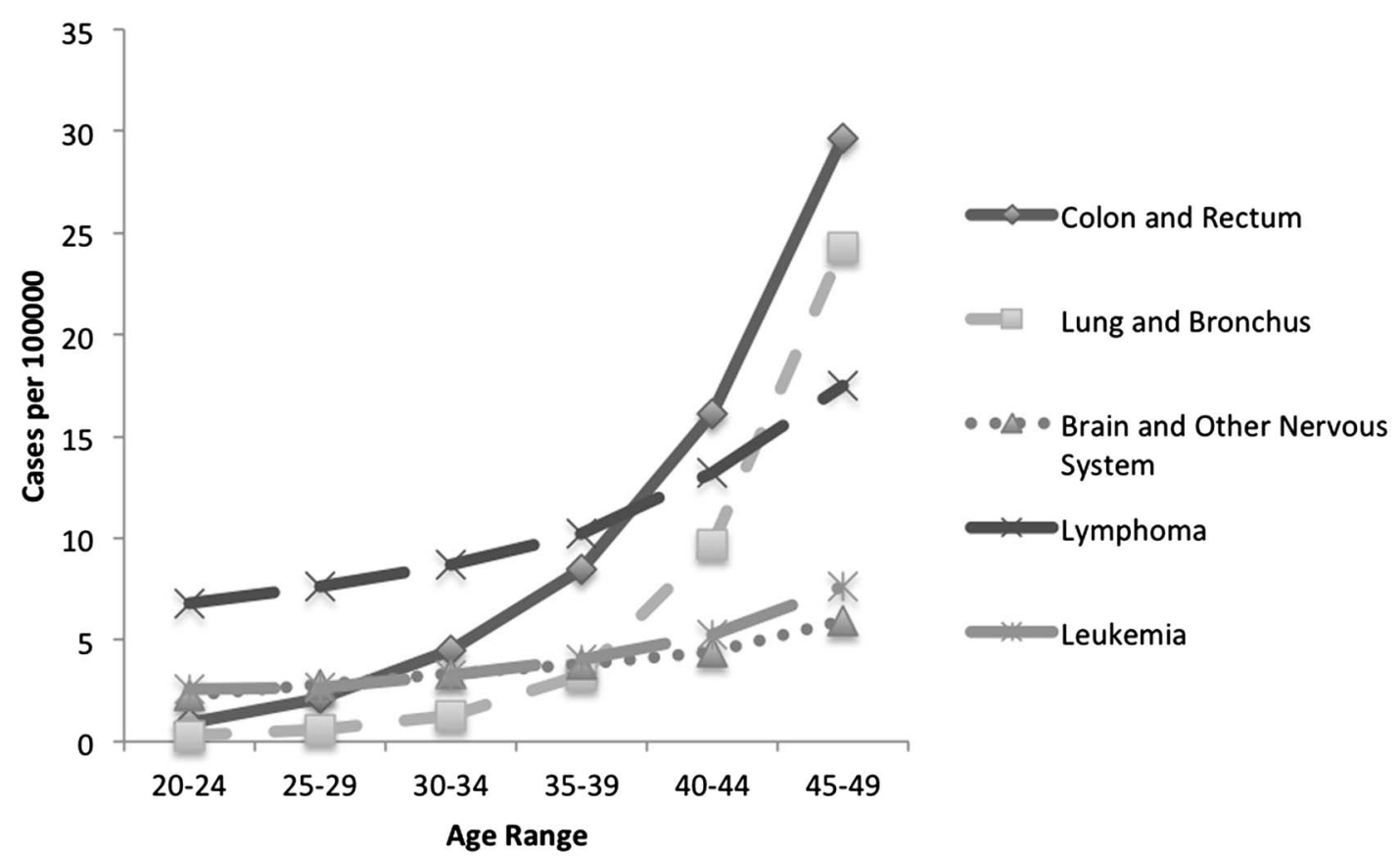

Figure 4 Age-specific incidence of the five most common cancers among young men and women combined, age 20-49, SEER 20012010.

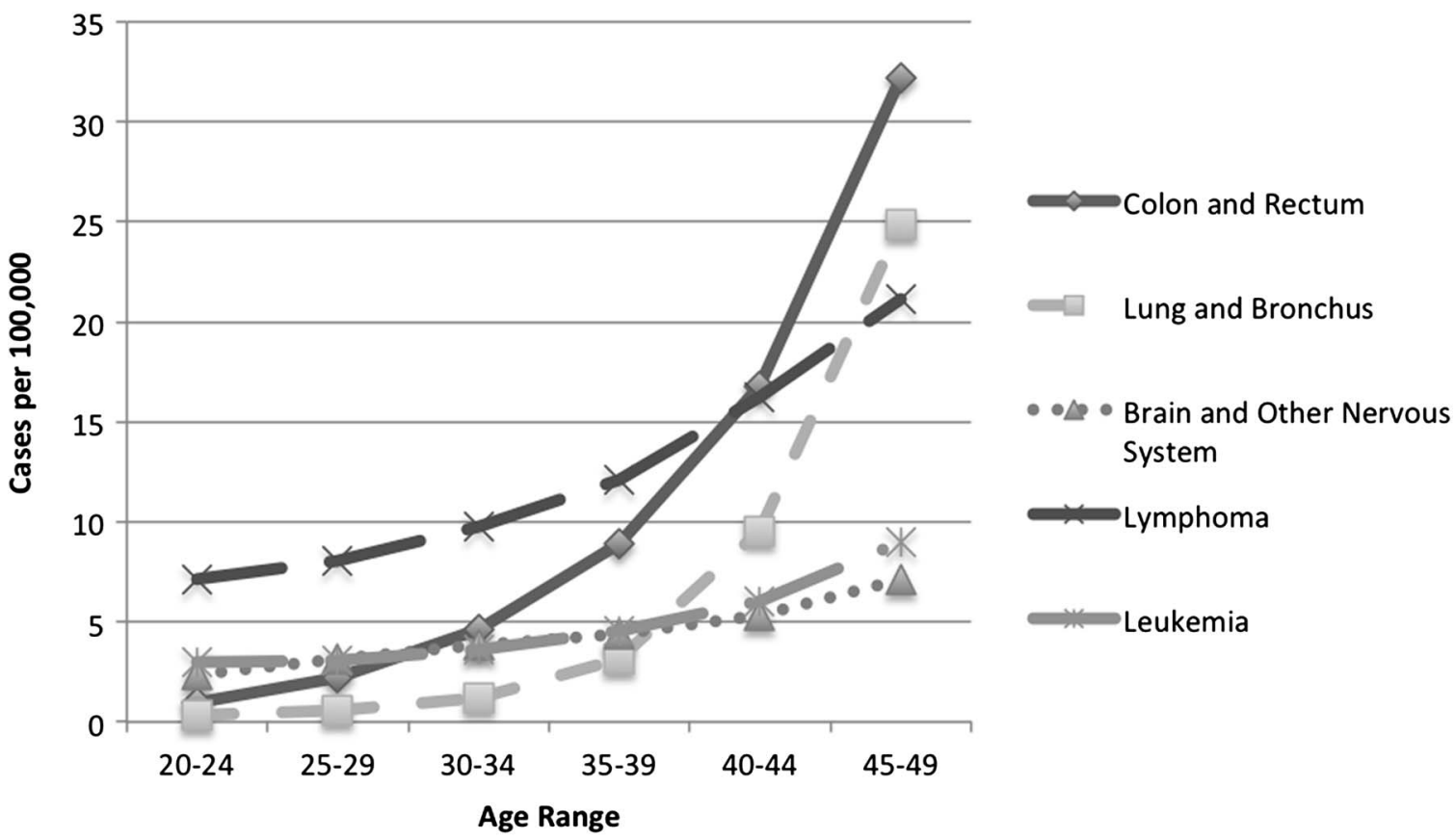

Figure 5 Age-specific incidence of the five most common cancers in young men age 20-49 years, SEER 2001-2010.

prudent as recommended by others, ${ }^{5}$ we believe reducing morbidity and mortality from young-onset CRC will require a more focused effort toward identifying individuals who will benefit from systematic screening. This is because individuals with signs/symptoms of CRC are already more likely to have advanced stage disease ${ }^{7}$ and it is likely that only a minority of young-onset CRC cases have an inherited CRC syndrome or would be identified by family history alone. ${ }^{8}$ Additionally, more epidemiologic work is needed to determine whether increasing rates of young-onset CRC are being observed in other countries around the world.

Given available screening technologies, population screening for individuals younger than age 50 is unlikely to 


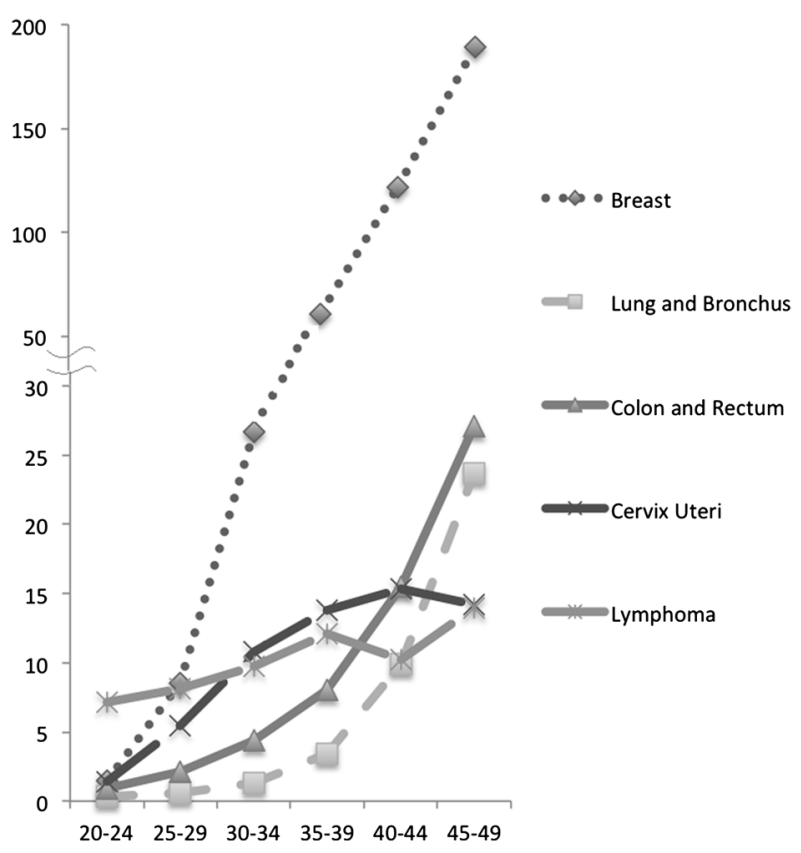

Figure 6 Age-specific incidence of the five most common cancers in young women age 20-49 years, SEER 2001-2010.

be efficient or cost-effective. However, we speculate that strategies for identifying high-risk individuals can be developed. For example, efforts might focus on identifying 4049-year-olds (who account for $75 \%$ of young-onset cancers) with CRC risk similar to individuals older than age 50. This might be accomplished by developing risk models that take into account demographic factors (eg, race/ethnicity), family history and genetics, constitutional factors (eg, high body mass index), and exposures (eg, smoking) associated with CRC risk.

Several limitations may be considered when interpreting our analysis. The SEER registry is wholly dependent on the conditions diagnosed and recorded by providers. If a condition is underdiagnosed or asymptomatic, as early cancer states are likely to be, reliance on this method may lead to reduced ascertainment. Additionally, the SEER registry does not provide any data on morbidity or the potential cancer risk factors described above. Such data might help clarify what subset of individuals under the age of 50 would be a reasonable target population for screening. Results are from analyses of US data-more work is required to determine whether the patterns observed are also occurring in other countries around the world.

In conclusion, we have demonstrated, using populationbased data, that CRC is a leading cause of cancer incidence and mortality among young adults in the USA. In context of trends toward increasing rates of CRC among young adults, strategies for identifying individuals at risk for young-onset CRC who might benefit from early age of screening initiation merit investigation.

Funding This study was funded (in part) by the National Cancer Institute, Comprehensive Partnerships to Advance Cancer Health Equity, grants \#U54CA132384 and \#U54CA132379 and by the Veteran's Affairs Health Services Research and Development under award number 5101 HX001574-02A1.

Competing interests None declared.

Provenance and peer review Not commissioned; externally peer reviewed.

\section{REFERENCES}

1 Torre LA, Bray F, Siegel RL, et al. Global cancer statistics, 2012. CA Cancer J Clin 2015;65:87-108.

2 Siegel RL, Jemal A, Ward EM. Increase in incidence of colorectal cancer among young men and women in the United States. Cancer Epidemiol Biomarkers Prev 2009;18:1695-8.

3 Edwards BK, Ward E, Kohler BA, et al. Annual report to the nation on the status of cancer, 1975-2006, featuring colorectal cancer trends and impact of interventions (risk factors, screening, and treatment) to reduce future rates. Cancer 2010;116:544-73.

4 You YN, Xing Y, Feig BW, et al. Young-onset colorectal cancer: is it time to pay attention? Arch Intern Med 2012;172:287-9.

5 Ahnen DJ, Wade SW, Jones WF, et al. The increasing incidence of young-onset colorectal cancer: a call to action. Mayo Clin Proc 2014;89:216-24.

6 Surveillance, Epidemiology, and End Results Program: Turning Cancer Data into Discovery. About the SEER Program. National Cancer Institute, n.d. Web. Fall. 2014. http://seer.cancer.gov/about/overview.html

7 Singh KE, Taylor TH, Pan CG, et al. Colorectal cancer incidence among young adults in California. J Adolesc Young Adult Oncol 2014;3:176-84.

8 Limburg PJ, Harmsen WS, Chen $\mathrm{HH}$, et al. Prevalence of alterations in DNA mismatch repair genes in patients with young-onset colorectal cancer. Clin Gastroenterol Hepatol 2011;9:497-502. 